\title{
Reduction of Feynman integrals in the parametric representation
}

\author{
Wen Chen \\ Department of Physics, University of Alberta, \\ Edmonton, AB T6G 2E1, Canada \\ E-mail: wchen1@ualberta.ca
}

ABSTRACT: In this paper, the reduction of Feynman integrals in the parametric representation is considered. This method proves to be more efficient than the integration-by-part (IBP) method in the momentum space. Tensor integrals can directly be parametrized without performing tensor reductions. The integrands of parametric integrals are functions of Lorentz scalars, instead of four momenta. The complexity of a calculation is determined by the number of propagators that are present rather than the number of all the linearly independent propagators. Furthermore, the symmetries of Feynman integrals under permutations of indices are transparent in the parametric representation. Since all the indices of the propagators are nonnegative, an algorithm to solve those identities can easily be developed, which can be used for automatic calculations.

KEYWORDS: NLO Computations, QCD Phenomenology

ARXIV EPRINT: 1902.10387 


\section{Contents}

1 Introduction 1

2 Identities between Feynman integrals in the parametric representation 2

3 The algorithm 3

4 Discussion 5

A Parametrization of tensor integrals 5

$\begin{array}{lr}\text { B Derivation of eq. (2.3) } & 8\end{array}$

\section{Introduction}

Nowadays high precision calculations are more and more important in high energy physics, which rely on calculations of multi-loop Feynman integrals. A standard strategy to calculate Feynman integrals is to reduce a large number of integrals to a smaller set of master integrals and then calculate the master integrals either analytically or numerically. A widely used technique to reduce Feynman integrals is the integration-by-part (IBP) method [1, 2]. Combining with various algorithms to solve IBP identities [3-9], IBP method proves to be very powerful in practice. Many published programs that implement these algorithms can be found [10-16].

Nevertheless, there are some disadvantages for the traditional IBP method. A Feynman integral is a function of Lorentz invariants, while the integrand is not. Thus the original Feynman integral contains redundant information, of which Lorentz-invariance identities [17] are direct consequences. In order to reduce the number of independent external momenta, one has to reduce tensor integrals into a set of scalar integrals before using the IBP technique. However, for tensors of high ranks, the reduction by itself is very complicated. Furthermore, in order to construct IBP identities, all the linearly independent propagators should be considered, though some of them may not be present in the integrals to be reduced.

These problems can be solved by considering Feynman integrals in the parametric representation. It was suggested in ref. [18] that IBP relations can be derived directly in the Lee-Pomeransky representation [19]. In this paper, a similar representation is used. It is shown that linear relations between integrals in this representation can directly be constructed. Feynman integrals can be reduced to master integrals by solving these linear relations, just as in the traditional IBP method. The advantage of this method is that it facilitates the reduction of tensor integrals (to be discussed in a subsequent paper [20]).

This paper is organized as follows. In section 2, identities between Feynman integrals in the parametric representation are constructed. The detail derivation of these identities can be found in appendix B. In section 3, we present a naive algorithm to solve these identities. 


\section{Identities between Feynman integrals in the parametric representation}

It is well-known that a dimensionally regularized $L$-loop Feynman integral with $n$ propagators can be parametrized by integrals of the following structure [21-23]:

$$
I=\frac{\Gamma\left(-\lambda_{f}\right)}{\prod_{i=1}^{n} \Gamma\left(\lambda_{i}+1\right)} \int d \Pi^{(n)} U^{\lambda_{u}} F^{\lambda_{f}} \prod_{i=1}^{n} x_{i}^{\lambda_{i}}, \quad \lambda \notin \mathbb{Z}^{-} .
$$

Here $d \Pi^{(n)} \equiv d x_{1} d x_{2} \cdots d x_{n} \delta\left(\sum_{i}\left|x_{i}\right|-1\right)$, where the sum in the delta function runs over any nontrivial subset of $\left\{x_{1}, x_{2}, \cdots x_{n}\right\}$. $U$ and $F$ are homogeneous polynomials of $x$ of degrees $L$ and $L+1$ respectively. By virtue of Mellin transformation, we may rewrite the integral in eq. (2.1) in the following form:

$$
\begin{aligned}
I & =\frac{\Gamma\left(-\lambda_{u}-\lambda_{f}\right)}{\Gamma\left(-\lambda_{u}\right) \prod_{i=1}^{n} \Gamma\left(\lambda_{i}+1\right)} \int d x_{n+1} d \Pi^{(n)}\left(U x_{n+1}+F\right)^{\lambda_{u}+\lambda_{f}} x_{n+1}^{-\lambda_{u}-1} \prod_{i=1}^{n} x_{i}^{\lambda_{i}} \\
& \equiv \frac{\Gamma\left(-\lambda_{0}\right)}{\prod_{i=1}^{n+1} \Gamma\left(\lambda_{i}+1\right)} \int d x_{n+1} d \Pi^{(n)} \mathcal{F}^{\lambda_{0}} \prod_{i=1}^{n+1} x_{i}^{\lambda_{i}}, \quad \lambda \notin \mathbb{Z}^{-} .
\end{aligned}
$$

It's easy to prove that the sum in the delta function in $d \Pi^{(n)}$ can be extended to include $x_{n+1}$. Thus we have

$$
I=\frac{\Gamma\left(-\lambda_{0}\right)}{\prod_{i=1}^{n+1} \Gamma\left(\lambda_{i}+1\right)} \int d \Pi^{(n+1)} \mathcal{F}^{\lambda_{0}} \prod_{i=1}^{n+1} x_{i}^{\lambda_{i}} \equiv \int d \Pi^{(n+1)} \mathcal{I}^{(-n-1)}, \quad \lambda \notin \mathbb{Z}^{-},
$$

where $\mathcal{I}^{(-n-1)}$ is a homogeneous function of $x$ of degree $-n-1$.

Tensor integrals can be parametrized by the standard procedure by parametrizing the denominators by Gauss integrals, shifting the loop momenta, and replacing loop momenta in the numerator by a sum of products of metric tensors. Alternatively, they can be parametrized by the generator method developed in appendix A (cf. eq. (A.8)).

According to the derivation in appendix B, we have the following identities:

$$
0=\int d \Pi^{(n+1)} \frac{\partial}{\partial x_{i}} \mathcal{I}^{(-n)}+\left.\delta_{\lambda_{i} 0} \int d \Pi^{(n)} \mathcal{I}^{(-n)}\right|_{x_{i}=0}, \quad i=1,2, \cdots, n+1, \lambda \notin \mathbb{Z}^{-},
$$

where $\delta_{\lambda_{i} 0}$ is the Kronecker delta. This equation can be understood as a generalization of IBP identities in the parametric representation $[18,19]$. To see this, we choose $d \Pi^{(n)}=\prod_{i=1}^{n+1} d x_{i} \delta\left(x_{n+1}-1\right)=\prod_{i=1}^{n} d x_{i}$. Then eq. (2.3) becomes

$$
\begin{aligned}
0 & =\int \prod_{j=1}^{n} d x_{j} \frac{\partial}{\partial x_{i}}\left[\prod_{j=1}^{n} x_{j}^{\lambda_{j}}\left(\left.\mathcal{F}\right|_{x_{n+1}=1}\right)^{\lambda_{0}}\right]+\delta_{\lambda_{i} 0} \int \prod_{j \neq i} d x_{j}\left(\left.\mathcal{F}\right|_{x_{i}=0, x_{n+1}=1}\right)^{\lambda_{0}} \prod_{j \neq i} x_{j}^{\lambda_{j}} \quad(2.4) \\
& \equiv \int \prod_{i=j}^{n} d x_{j} \frac{\partial}{\partial x_{i}}\left[\prod_{j=1}^{n} x_{j}^{\lambda_{j}} \mathcal{G}^{\lambda_{0}}\right]+\delta_{\lambda_{i} 0} \int \prod_{j \neq i} d x_{j}\left(\left.\mathcal{G}\right|_{x_{i}=0}\right)^{\lambda_{0}} \prod_{j \neq i} x_{j}^{\lambda_{j}}, \quad i=1,2, \cdots, n, \lambda \in \mathbb{Z}^{-},
\end{aligned}
$$

which are exactly the IBP identities in the Lee-Pomeransky representation (except for that here we don't consider the cases where $\lambda \notin \mathbb{Z}^{-}$, which is unnecessary in practice). 
Among identities eq. (2.3), some are recurrence relations between integrals with shifted dimensions [5,24]. Linear relations free of dimensional recurrence can be derived by using the method of parametric annihilators $[3,18,19,25]$ or the syzygy-equation method [26-28]. ${ }^{1}$ For our approach, we do need these dimensional-recurrence relations, because tensor integrals are parametrized by integrals with shifted dimensions.

By applying eq. (2.3), integrals associated with subdiagrams may arise, some of which may be scaleless. Scaleless integrals can be identified by the criterion that equation

$$
\sum_{i=1}^{n} k_{i} x_{i} \frac{\partial \mathcal{G}}{\partial x_{i}}=\mathcal{G}
$$

has a nontrivial $x$-independent solution for $k[29]$.

\section{The algorithm}

In this section, we give a brief description of the algorithm we use to solve the linear relations in eq. (2.3). We use an algorithm similar to that in ref. [5] combined with the application of symbolic rules [13].

An ordering for the integrals is prescribed. Integrals of the highest priority is solved first. By fixing the values of $\lambda$, eq. (2.3) can be solved symbolically. These solutions play the role of symbolic rules. For one-loop integrals, these symbolic rules are complete, in the sense that any one-loop integral can be reduced to master integrals by applying these rules. Thus the reduction of one-loop integrals is extremely fast by using this algorithm. While for multi-loop integrals, these symbolic rules are incomplete. In this case, we have to reduce the unreduced integrals integrals by solving eq. (2.3) with the explicit values of $\lambda$ substituted in. These identities can be solved by Gauss elimination.

For convenience, we may express a parametric integral by a standard Feynman integral defined in dimension $-2 \lambda_{0} \equiv d+m$, where $d$ is the space-time dimension and $m$ is a nonnegative integer. This kind of integrals can be numerically evaluated by using FIESTA [30]. Since eq. (2.3) contains dimensional-recurrence relations, in order the process to terminate, we use an explicit cut-off: $m \geq 0$.

As a trivial example, we consider the reduction of the tadpole integral:

$$
\begin{aligned}
I\left(\lambda_{0}, \lambda_{1}\right) & \equiv(-1)^{\lambda_{1}} i \pi^{\lambda_{0}} \int d^{-2 \lambda_{0}} l \frac{1}{\left(l^{2}-m^{2}\right)^{1+\lambda_{1}}} \\
& =\frac{\Gamma\left(-\lambda_{0}\right)}{\Gamma\left(\lambda_{1}+1\right) \Gamma\left(\lambda_{2}+1\right)} \int d \Pi^{(2)}\left(m^{2} x_{1}^{2}+x_{1} x_{2}\right)^{\lambda_{0}} x_{1}^{\lambda_{1}} x_{2}^{\lambda_{2}},
\end{aligned}
$$

where $\lambda_{2}=-2 \lambda_{0}-\lambda_{1}-2$. By applying eq. (2.3), we get following linear relations:

$$
\begin{aligned}
& 0=I\left(\lambda_{0}, \lambda_{1}\right)-\left(\lambda_{1}+1\right) I\left(\lambda_{0}-1, \lambda_{1}+1\right), \\
& 0=\left(2 \lambda_{0}+\lambda_{1}\right) I\left(\lambda_{0}-1, \lambda_{1}\right)-2 m^{2}\left(\lambda_{1}+1\right) I\left(\lambda_{0}-1, \lambda_{1}+1\right)+I\left(\lambda_{0}, \lambda_{1}-1\right) .
\end{aligned}
$$

Here it is understood that

$$
I\left(\lambda_{0},-1\right)=\frac{\Gamma\left(-\lambda_{0}\right)}{\Gamma\left(\lambda_{2}+1\right)} \int d \Pi^{(1)}\left(\left.\left(m^{2} x_{1}^{2}+x_{1} x_{2}\right)\right|_{x_{1}=0}\right)^{\lambda_{0}} x_{2}^{\lambda_{2}}=0 .
$$

\footnotetext{
${ }^{1}$ The latter method is based on Baikov representation [3]. Nevertheless, it can easily be applied to the representation used in this paper.
} 
These linear relations can be solved symbolically. The solutions read

$$
\begin{aligned}
& I\left(\lambda_{0}, \lambda_{1}\right)=\frac{1}{\lambda_{1}} I\left(\lambda_{0}+1, \lambda_{1}-1\right), \quad \lambda_{1} \geq 1, \\
& I\left(\lambda_{0}, \lambda_{1}\right)=\frac{2 m^{2}}{2 \lambda_{0}+\lambda_{1}+2} I\left(\lambda_{0}+1, \lambda_{1}\right)-\frac{1}{2 \lambda_{0}+\lambda_{1}+2} I\left(\lambda_{0}+1, \lambda_{1}-1\right) .
\end{aligned}
$$

These solutions play the role of symbolic rules. Obviously these rules are complete in this example. The first rule can be used to reduce the index $\lambda_{1}$, and the second rule can be used to reduce the spacetime dimension.

As a less trivial example, we consider the reduction of the following two-loop massless double-box integral:

$$
M^{\mu \nu}=\int d^{d} l_{1} d^{d} l_{2} P(-1,-1,-1,-1,-1,-1,-1) l_{1}^{\mu} l_{2}^{\nu},
$$

where

$$
P\left(i_{1}, i_{2}, i_{3}, i_{4}, i_{5}, i_{6}, i_{7}\right) \equiv l_{1}^{2 i_{1}} l_{2}^{2 i_{2}}\left(l_{1}+k_{1}\right)^{2 i_{3}}\left(l_{1}-k_{2}\right)^{2 i_{4}}\left(l_{1}+l_{2}+k_{1}\right)^{2 i_{5}}\left(l_{1}+l_{2}-k_{2}\right)^{2 i_{6}}\left(l_{1}+l_{2}-k_{2}-k_{3}\right)^{2 i_{7}} .
$$

We put $\left(k_{1}+k_{2}\right)^{2}=8$, and $\left(k_{1}+k_{3}\right)^{2}=-1$. By expressing parametric integrals in terms of standard Feynman integrals, the result reads:

$$
\begin{aligned}
& M^{\mu \nu}=\frac{1}{16 d-58}\left[7(d-3) k_{1}^{\mu} k_{2}^{\nu}+(d-4) k_{2}^{\mu} k_{2}^{\nu}+8(d-4) k_{3}^{\mu} k_{2}^{\nu}+7(d-11) k_{1}^{\mu} k_{3}^{\nu}+(d-11) k_{2}^{\mu} k_{3}^{\nu}\right. \\
& \left.\quad+8(d-11) k_{3}^{\mu} k_{3}^{\nu}-28 g^{\mu \nu}-49 k_{1}^{\mu} k_{1}^{\nu}-56 k_{3}^{\mu} k_{1}^{\nu}\right] \int d^{d} l_{1} d^{d} l_{2} P(-1,-1,-1,-1,-1,-1,-1) \\
& \quad+\frac{d-3}{8 d-29}\left[\frac{1}{8}(3 d-14) g^{\mu \nu}+\frac{7}{64}(1-2 d) k_{1}^{\mu} k_{1}^{\nu}+\frac{1}{64}(29-8 d) k_{2}^{\mu} k_{1}^{\nu}+\frac{1}{64}\left(-68 d^{2}+10 d+119\right) k_{1}^{\mu} k_{2}^{\nu}\right. \\
& \quad+\frac{1}{448}\left(-62 d^{2}+10 d+91\right) k_{2}^{\mu} k_{2}^{\nu}+\frac{1}{8}(1-2 d) k_{3}^{\mu} k_{1}^{\nu}+\frac{1}{56}\left(-62 d^{2}+10 d+91\right) k_{3}^{\mu} k_{2}^{\nu} \\
& \left.\quad+\frac{1}{32}\left(5 d^{2}-38 d+49\right) k_{1}^{\mu} k_{3}^{\nu}+\frac{1}{224}\left(5 d^{2}-38 d+49\right) k_{2}^{\mu} k_{3}^{\nu}+\frac{1}{28}\left(5 d^{2}-38 d+49\right) k_{3}^{\mu} k_{3}^{\nu}\right] \\
& \quad \times \int d^{d+2} l_{1} d^{d+2} l_{2} P(-1,-1,-1,-1,-1,-1,-1)+\ldots,
\end{aligned}
$$

where the ellipsis represents contributions of subdiagrams, which are too complicated to be presented here. All the rest master integrals are

$$
\begin{aligned}
& \int d^{d} l_{1} d^{d} l_{2} P(-1,-1,0,0,0,0,-1), \\
& \int d^{d} l_{1} d^{d} l_{2} P(0,-1,-1,0,0,-1,0), \\
& \int d^{d} l_{1} d^{d} l_{2} P(-1,-1,0,0,-1,-1,0), \\
& \int d^{d} l_{1} d^{d} l_{2} P(0,0,-1,-1,-1,-1,0), \\
& \int d^{d} l_{1} d^{d} l_{2} P(-1,-1,-1,-1,0,0,-1), \\
& \int d^{d} l_{1} d^{d} l_{2} P(-1,-1,0,-1,-1,0,-1) .
\end{aligned}
$$


The calculation is carried out by using a private code. To check the calculation, we contract the tensor integral with some external momenta, and reduce the resulting integral by using FIRE [16]. The basis chosen by FIRE contains an integral with a double propagator. This integral can be further reduced by using our code. The final result for the tensor integral thus obtained is consistent with the one obtained by using our code. For integrals with simpler topologies, we have also verified the calculations numerically by using FIESTA [30] (Notice that FIESTA can be used to evaluate integrals with numerators and with shifted dimensions).

\section{Discussion}

In this paper, the reduction of Feynman integrals in the parametric representation is considered. A representation similar to the Lee-Pomeransky representation is used. Tensor integrals can directly be parametrized by using a generator method. Identities between the parametric integrals are derived. Feynman integrals are reduced to master integrals by solving these identities. This method has many advantages over the traditional IBP technique, as is discussed at the very beginning of this paper. Symbolic rules can be derived out of these identities. One-loop integrals can be reduced to master integrals merely by applying these rules. For multi-loop integrals, these rules are incomplete. Thus we can not get rid of the Gauss elimination, which is less efficient. Though the symbolic rules are incomplete, the reduction is as complete as that of the traditional IBP method in the sense that numbers of master integrals obtained by these two methods are the same.

\section{Acknowledgments}

The author thanks Shuai Liu for testing the code. This work was supported by the Natural Sciences and Engineering Research Council of Canada.

\section{A Parametrization of tensor integrals}

It is well-known that a propagator $\frac{1}{D_{i}} \equiv \frac{1}{p_{i}^{2}-m_{i}^{2}+i \delta}$ can be parameterized by

$$
\frac{1}{D_{i}^{\lambda_{i}+1}}=\frac{e^{-\frac{\lambda_{i}+1}{2} i \pi}}{\Gamma\left(\lambda_{i}+1\right)} \int_{0}^{\infty} d x_{i} e^{i x_{i} D_{i}} x_{i}^{\lambda_{i}}, \quad \operatorname{Im}\left\{D_{i}\right\}>0 .
$$

A cut propagator can be parametrized similarly:

$$
-2 \pi i \delta\left(D_{i}\right)=e^{-\frac{1}{2} \pi i} \int_{-\infty}^{\infty} d x_{i} e^{i x_{i} D_{i}}
$$

By virtue of the identity

$$
l_{i_{1}}^{\mu_{1}} l_{i_{2}}^{\mu_{2}} \cdots l_{i_{m}}^{\mu_{m}}=\frac{i(-1)^{m}}{\Gamma(m+1)}\left[\frac{\partial}{\partial p_{i_{1}, \mu_{1}}} \frac{\partial}{\partial p_{i_{2}, \mu_{2}}} \cdots \frac{\partial}{\partial p_{i_{m}, \mu_{m}}} \int_{0}^{\infty} d y \exp \left[-i y\left(1+\sum_{i=1}^{L} p_{i} \cdot l_{i}\right)\right]\right]_{p_{i}^{\mu}=0},
$$


a $d$ dimensional $L$-loop rank $m$ tensor integral with $n$ propagators can be generated by

$$
\begin{aligned}
& \mathcal{M}^{\mu_{1} \mu_{2} \cdots \mu_{m}}\left(d, \lambda_{1}, \lambda_{2}, \cdots, \lambda_{n}\right) \\
& \quad \equiv \pi^{-L d / 2} \int d^{d} l_{1} d^{d} l_{2} \cdots d^{d} l_{L} \frac{l_{i_{1}}^{\mu_{1}} l_{i_{2}}^{\mu_{2}} \cdots l_{i_{m}}^{\mu_{m}}}{D_{1}^{\lambda_{1}+1} D_{2}^{\lambda_{2}+1} \cdots D_{n}^{\lambda_{n}+1}} \\
& \quad=\frac{(-1)^{m}}{\Gamma(m+1)}\left[\frac{\partial}{\partial p_{i_{1}, \mu_{1}}} \frac{\partial}{\partial p_{i_{2}, \mu_{2}}} \cdots \frac{\partial}{\partial p_{i_{m}, \mu_{m}}} \mathcal{M}_{p}\left(d, \lambda_{1}, \lambda_{2}, \cdots, \lambda_{n}\right)\right]_{p_{i}^{\mu}=0}
\end{aligned}
$$

where $d_{0}$ is the real space-time dimension, and the generator

$$
\begin{aligned}
& \mathcal{M}_{p}\left(d, \lambda_{1}, \lambda_{2}, \cdots, \lambda_{n}\right) \\
& \equiv i \pi^{-L d / 2} \int d y \prod_{i=1}^{n}\left(d x_{i} \frac{x_{i}^{\lambda_{i}} e^{-\frac{i \pi}{2}\left(\lambda_{i}+1\right)}}{\Gamma\left(\lambda_{i}+1\right)}\right) \int \prod_{j=1}^{L} d^{d} l_{j} \exp \left[i \sum_{i=1}^{n} x_{i} D_{i}-i y\left(1+\sum_{i=1}^{L} p_{i} \cdot l_{i}\right)\right] \\
& \equiv i \pi^{-L d / 2} \int d y \prod_{i=1}^{n}\left(d x_{i} \frac{x_{i}^{\lambda_{i}} e^{-\frac{i \pi}{2}\left(\lambda_{i}+1\right)}}{\Gamma\left(\lambda_{i}+1\right)}\right) \int \prod_{j=1}^{L} d^{d} l_{j} \exp \left[i\left(\sum_{i, j=1}^{L} A_{i j} l_{i} \cdot l_{j}+2 \sum_{i=1}^{L} B_{i} \cdot l_{i}+C\right)\right] \\
& =s_{g}^{-L / 2} e^{\frac{i \pi}{2}\left(\frac{L d}{2}+1\right)} \int d y \prod_{i=1}^{n}\left(d x_{i} \frac{x_{i}^{\lambda_{i}} e^{-\frac{i \pi}{2}\left(\lambda_{i}+1\right)}}{\Gamma\left(\lambda_{i}+1\right)}\right) \operatorname{det}(A)^{-\frac{d}{2}} \exp \left[i\left(C-\sum_{i, j}^{L}\left(A^{-1}\right)_{i j} B_{i} \cdot B_{j}\right)\right] .
\end{aligned}
$$

Here $s_{g}$ is the determinant of the dimensionally regularized spacetime metric. For instance, in four dimensional Minkowski spacetime, we have $s_{g}=e^{i \pi(d-1)}$, and in four dimensional Euclidean space, we have $s_{g}=e^{i \pi(d-4)}$ (instead of 1 ). To simplify the above integral, we insert a trivial integral $\int_{0}^{\infty} d \alpha(\alpha-E(x))$ into it, where $E(x)$ is a positive homogeneous function of $x_{i}$ 's of degree 1 . In this paper, we choose $E(x)=\sum_{i}\left|x_{i}\right|$, where the sum in the delta function runs over any nontrivial subset of $\left\{x_{1}, x_{2}, \cdots x_{n}\right\}$. It should be noticed that $E(x)$ need not to be linear in $x$ 's. Rescaling the variables of integration by $x_{i} \rightarrow \alpha x_{i}$, and $y \rightarrow \alpha y$, and integrating over $\alpha$, we get

$$
\begin{aligned}
& \mathcal{M}_{p}\left(d, \lambda_{1}, \lambda_{2}, \cdots, \lambda_{n}\right) \\
= & s_{g}^{-L / 2} e^{i \pi \lambda_{f}} \frac{\Gamma\left(1-\lambda_{f}\right)}{\prod_{i=1}^{n} \Gamma\left(\lambda_{i}+1\right)} \int d y \prod_{i=1}^{n}\left(d x_{i} x_{i}^{\lambda_{i}}\right) \delta(1-E(x)) U^{1-\frac{d}{2}-\lambda_{f}} F(p, y)^{\lambda_{f}-1} \\
= & s_{g}^{-L / 2} e^{i \pi \lambda_{f}} \frac{\Gamma\left(\frac{d}{2}\right)}{\Gamma\left(\lambda_{f}+\frac{d}{2}-1\right) \prod_{i=1}^{n} \Gamma\left(\lambda_{i}+1\right)} \\
& \times \int d y \prod_{i=1}^{n+1} d x_{i} \delta(1-E(x)) \mathcal{F}(p, y)^{-\frac{d}{2}} x_{n+1}^{\lambda_{f}+\frac{d}{2}-2} \prod_{i=1}^{n} x_{i}^{\lambda_{i}} \\
\equiv & s_{g}^{-L / 2} e^{i \pi \lambda_{f}} \frac{\Gamma\left(\frac{d}{2}\right)}{\Gamma\left(\lambda_{n+1}\right) \prod_{i=1}^{n} \Gamma\left(\lambda_{i}+1\right)} \int d y d \Pi^{(n+1)} \mathcal{F}(p, y)^{-\frac{d}{2}} x_{n+1}^{\lambda_{n+1}-1} \prod_{i=1}^{n} x_{i}^{\lambda_{i}},
\end{aligned}
$$

where $\lambda_{f} \equiv \frac{1}{2} d L-n-\sum_{i=1}^{n} \lambda_{i}, U \equiv \operatorname{det}(A), F(p, y) \equiv U\left(\sum_{i, j}^{L}\left(A^{-1}\right)_{i j} B_{i} \cdot B_{j}-C\right)$, $\mathcal{F}(p, y) \equiv U x_{n+1}+F(p, y)$, and $d \Pi^{(n)}$ is the one defined in eq. (2.1). 
Generally, $\mathcal{F}(p, y)$ is of the form

$$
\begin{aligned}
\mathcal{F}(p, y) & =\mathcal{F}(0,0)+y U-y U \sum_{i, j=1}^{L}\left[\left(A^{-1}\right)_{i j} B_{i} \cdot p_{j}\right]_{y=0}+\frac{1}{4} y^{2} U \sum_{i, j=1}^{L}\left[\left(A^{-1}\right)_{i j} p_{i} \cdot p_{j}\right]_{y=0} \\
& \equiv \mathcal{F}(0,0)+y U+y \sum_{i=1}^{L} b_{i} \cdot p_{i}+\sum_{i, j}^{L} c_{i j} y^{2} p_{i} \cdot p_{j},
\end{aligned}
$$

where $b$ and $c$ are polynomials of $x$ 's. That is $b_{i}^{\mu}=b_{i}^{\mu}\left(x_{1}, x_{2}, \cdots, x_{n}\right)$, and $c_{i j}=c_{i j}\left(x_{1}, x_{2}\right.$, $\cdots, x_{n}$ ). Setting $p^{\mu}=0$, the integration over $y$ in eq. (A.2) can easily be carried out by shifting the variable $x_{n+1} \rightarrow x_{n+1}-y$.

$$
\begin{aligned}
\mathcal{M}_{0}\left(d, \lambda_{1}, \lambda_{2}, \cdots, \lambda_{n}\right)= & s_{g}^{-L / 2} e^{i \pi \lambda_{f}} \frac{\Gamma\left(\frac{d}{2}\right)}{\Gamma\left(\lambda_{n+1}\right) \prod_{i=1}^{n} \Gamma\left(\lambda_{i}+1\right)} \\
& \times \int_{0}^{\infty} d x_{n+1} \int_{0}^{x_{n+1}} d y \int d \Pi^{(n)} \mathcal{F}(0,0)^{-\frac{d}{2}}\left(x_{n+1}-y\right)^{\lambda_{n+1}-1} \prod_{i=1}^{n} x_{i}^{\lambda_{i}} \\
= & s_{g}^{-L / 2} e^{i \pi \lambda_{f}} \frac{\Gamma\left(\frac{d}{2}\right)}{\prod_{i=1}^{n+1} \Gamma\left(\lambda_{i}+1\right)} \int d \Pi^{(n+1)} \mathcal{F}(0,0)^{-\frac{d}{2}} \prod_{i=1}^{n+1} x_{i}^{\lambda_{i}} \\
\equiv & s_{g}^{-L / 2} e^{i \pi \lambda_{f}} \int d \Pi^{(n+1)} \mathcal{I}\left(\lambda_{0}, \lambda_{1}, \cdots, \lambda_{n}\right) \\
\equiv & s_{g}^{-L / 2} e^{i \pi \lambda_{f}} I\left(\lambda_{0}, \lambda_{1}, \cdots, \lambda_{n}\right)
\end{aligned}
$$

Similarly we have:

$$
\frac{\Gamma\left(\frac{d}{2}\right)}{\Gamma\left(\lambda_{n+1}-\delta_{y}\right) \prod_{i=1}^{n} \Gamma\left(\lambda_{i}+1\right)} \int d y d \Pi^{(n+1)} \mathcal{F}(0, y)^{-\frac{d}{2}} y^{\delta_{y}} x_{n+1}^{\lambda_{n+1}-\delta_{y}-1} \prod_{i=1}^{n} x_{i}^{\lambda_{i}}=\Gamma\left(\delta_{y}+1\right) I\left(\lambda_{0}, \lambda_{1}, \cdots, \lambda_{n}\right) .
$$

And obviously we have

$$
\begin{aligned}
& \frac{\Gamma\left(\frac{d}{2}\right)}{\prod_{i=1}^{n+1} \Gamma\left(\lambda_{i}+1\right)} \int d \Pi^{(n)} x_{i}^{\delta_{i}} \mathcal{F}(0,0)^{-\frac{d}{2}} \prod_{j=1}^{n+1} x_{j}^{\lambda_{j}} \\
& =\prod_{j=1}^{\delta_{i}}\left(\lambda_{i}+j\right) I\left(d, \lambda_{1}, \lambda_{2}, \cdots, \lambda_{i}+\delta_{i}, \cdots, \lambda_{n}\right) \\
& =R_{i}^{\delta_{i}} I\left(-\frac{d}{2}, \lambda_{1}, \lambda_{2}, \cdots, \lambda_{n}\right),
\end{aligned}
$$

where $R_{i}$ is an operator applying on $I$ such that $R_{i} I\left(\lambda_{0}, \lambda_{1}, \cdots, \lambda_{i}, \cdots, \lambda_{n}\right) \equiv\left(\lambda_{i}+1\right) I\left(\lambda_{0}, \lambda_{1}\right.$, $\left.\cdots, \lambda_{i}+1, \cdots, \lambda_{n}\right)$. Similarly we define $D_{i} I\left(\lambda_{0}, \lambda_{1}, \cdots, \lambda_{i}, \cdots, \lambda_{n}\right) \equiv I\left(\lambda_{0}, \lambda_{1}, \cdots, \lambda_{i}-1, \cdots, \lambda_{n}\right)$. We define the operator

$$
P_{i, \mu} \equiv-\frac{\partial}{\partial p_{i}^{\mu}}+\left[b_{i, \mu}\left(R_{1}, R_{2}, \cdots, R_{n}\right)+2 \sum_{j=1}^{L} p_{j, \mu} c_{i j}\left(R_{1}, R_{2}, \cdots, R_{n}\right)\right] D_{0}
$$


where $b$ and $c$ are defined in eq. (A.3). Then by virtue of eqs. (A.1) to (A.3), (A.5) and (A.6), it's easy to see that

$$
\mathcal{M}^{\mu_{1} \mu_{2} \cdots \mu_{m}}\left(d, \lambda_{1}, \lambda_{2}, \cdots, \lambda_{n}\right)=s_{g}^{-L / 2} e^{i \pi \lambda_{f}}\left[P_{i_{1}}^{\mu_{1}} P_{i_{2}}^{\mu_{2}} \cdots P_{i_{m}}^{\mu_{m}} I\left(-\frac{d}{2}, \lambda_{1}, \lambda_{2}, \cdots, \lambda_{n}\right)\right]_{p^{\mu}=0} .
$$

Notice that the factor of $\frac{1}{\Gamma(m+1)}$ in eq. (A.1) is canceled by the factor $\Gamma\left(\delta_{y}+1\right)$ in eq. (A.5), because after applying the differential operators to $\mathcal{M}_{p}$ and putting $p^{\mu}=0$, the degree in $y$ of the integrand in eq. (A.2) is exactly $m$.

\section{B Derivation of eq. (2.3)}

By virtue of the homogeneity of the integrand $\mathcal{I}^{(-n-1)}$ in eq. (2.2), rescaling of the variables of integration leads to

$$
\begin{aligned}
\int d \Pi^{(n+1)} \mathcal{F}^{\lambda_{0}} \prod_{i=1}^{n+1} x_{i}^{\lambda_{i}} & =\int d x_{1} d x_{2} \cdots d x_{n+1} \delta\left(x_{n+1} / \alpha-1\right) \mathcal{F}^{\lambda_{0}} \prod_{i=1}^{n+1} x_{i}^{\lambda_{i}} \\
& =\left.\int d x_{1} d x_{2} \cdots d x_{n} \mathcal{F}^{\lambda_{0}}\right|_{x_{n+1}=\alpha} \alpha^{\lambda_{n+1}+1} \prod_{i=1}^{n} x_{i}^{\lambda_{i}} \\
& \equiv G(\alpha), \quad \lambda \notin \mathbb{Z}^{-} .
\end{aligned}
$$

Obviously $G(\alpha)$ should be independent of $\alpha$. Thus we have

$$
\begin{aligned}
0 & =\alpha \frac{\partial G(\alpha)}{\partial \alpha} \\
& =\int d x_{1} d x_{2} \cdots d x_{n} \alpha \frac{\partial}{\partial \alpha}\left[\left.\mathcal{F}^{\lambda_{0}}\right|_{x_{n+1}=\alpha} \alpha^{\lambda_{n+1}+1} \prod_{i=1}^{n} x_{i}^{\lambda_{i}}\right] \\
& =\int d x_{1} d x_{2} \cdots d x_{n+1} \delta\left(x_{n+1} / \alpha-1\right) \frac{\partial}{\partial x_{n+1}}\left[\mathcal{F}^{\lambda_{0}} x_{n+1}^{\lambda_{n+1}+1} \prod_{i=1}^{n} x_{i}^{\lambda_{i}}\right] \\
& =\int d \Pi^{(n+1)} \frac{\partial}{\partial x_{n+1}}\left[\mathcal{F}^{\lambda_{0}} x_{n+1}^{\lambda_{n+1}+1} \prod_{i=1}^{n} x_{i}^{\lambda_{i}}\right], \quad \lambda \notin \mathbb{Z}^{-} .
\end{aligned}
$$

Since $\lambda \notin \mathbb{Z}^{-}, \lambda_{n+1}+1 \neq 0$. Replacing $\lambda_{n+1}+1$ by $\lambda_{n+1}$, we get

$$
0=\int d \Pi^{(n+1)} \frac{\partial}{\partial x_{n+1}} \mathcal{I}^{(-n)}, \quad \lambda_{n+1} \neq 0, \lambda \notin \mathbb{Z}^{-} .
$$

Similarly, for a general $x_{i}$, we have

$$
0=\int d \Pi^{(n+1)} \frac{\partial}{\partial x_{i}} \mathcal{I}^{(-n)}, \quad \lambda_{i} \neq 0, \lambda \notin \mathbb{Z}^{-} .
$$

It's easy to prove that this equation still holds in the case where the domain of integration of $x_{i}$ is $(-\infty, \infty)$. 
We consider the limit $\lambda_{i} \rightarrow 0$. By using the formula $\frac{1}{x^{1-\lambda}}=\frac{1}{\lambda} \delta(x)+\mathcal{O}\left(\lambda^{0}\right)[31]$, we get

$$
\begin{aligned}
0 & =\lim _{\lambda_{i} \rightarrow 0} \int d \Pi^{(n+1)} \frac{\partial}{\partial x_{i}}\left[\mathcal{F}^{\lambda_{0}} x_{i}^{\lambda_{i}} \prod_{j \neq i}^{n+1} x_{j}^{\lambda_{j}}\right] \\
& =\int d \Pi^{(n+1)} \frac{\partial}{\partial x_{i}}\left[\mathcal{F}^{\lambda_{0}} \prod_{j \neq i}^{n+1} x_{j}^{\lambda_{j}}\right]+\left.\int d \Pi^{(n)} \mathcal{F}^{\lambda_{0}}\right|_{x_{i}=0} \prod_{j \neq i}^{n+1} x_{j}^{\lambda_{j}}, \quad \lambda \notin \mathbb{Z}^{-} .
\end{aligned}
$$

Then we have

$$
0=\int d \Pi^{(n+1)} \frac{\partial}{\partial x_{i}} \mathcal{I}^{(-n)}+\left.\int d \Pi^{(n)} \mathcal{I}^{(-n)}\right|_{x_{i}=0}, \quad \lambda_{i}=0, \lambda \notin \mathbb{Z}^{-} .
$$

Open Access. This article is distributed under the terms of the Creative Commons Attribution License (CC-BY 4.0), which permits any use, distribution and reproduction in any medium, provided the original author(s) and source are credited.

\section{References}

[1] F.V. Tkachov, A Theorem on Analytical Calculability of Four Loop Renormalization Group Functions, Phys. Lett. 100B (1981) 65 [INSPIRE].

[2] K.G. Chetyrkin and F.V. Tkachov, Integration by Parts: The Algorithm to Calculate B-functions in 4 Loops, Nucl. Phys. B 192 (1981) 159 [INSPIRE].

[3] P.A. Baikov, Explicit solutions of the multiloop integral recurrence relations and its application, Nucl. Instrum. Meth. A 389 (1997) 347 [hep-ph/9611449] [INSPIRE].

[4] O.V. Tarasov, Reduction of Feynman graph amplitudes to a minimal set of basic integrals, Acta Phys. Polon. B 29 (1998) 2655 [hep-ph/9812250] [INSPIRE].

[5] S. Laporta, High precision calculation of multiloop Feynman integrals by difference equations, Int. J. Mod. Phys. A 15 (2000) 5087 [hep-ph/0102033] [inSPIRE].

[6] A.V. Smirnov and V.A. Smirnov, Applying Grobner bases to solve reduction problems for Feynman integrals, JHEP 01 (2006) 001 [hep-lat/0509187] [INSPIRE].

[7] R.N. Lee, Group structure of the integration-by-part identities and its application to the reduction of multiloop integrals, JHEP 07 (2008) 031 [arXiv:0804.3008] [INSPIRE].

[8] P. Kant, Finding Linear Dependencies in Integration-By-Parts Equations: A Monte Carlo Approach, Comput. Phys. Commun. 185 (2014) 1473 [arXiv:1309.7287] [INSPIRE].

[9] A. von Manteuffel and R.M. Schabinger, A novel approach to integration by parts reduction, Phys. Lett. B 744 (2015) 101 [arXiv: 1406.4513] [InSPIRE].

[10] C. Anastasiou and A. Lazopoulos, Automatic integral reduction for higher order perturbative calculations, JHEP 07 (2004) 046 [hep-ph/0404258] [INSPIRE].

[11] A.V. Smirnov, Algorithm FIRE - Feynman Integral REduction, JHEP 10 (2008) 107 [arXiv: 0807.3243] [INSPIRE].

[12] C. Studerus, Reduze-Feynman Integral Reduction in $C++$, Comput. Phys. Commun. 181 (2010) 1293 [arXiv:0912.2546] [INSPIRE]. 
[13] R.N. Lee, Presenting LiteRed: a tool for the Loop InTEgrals REDuction, arXiv:1212.2685 [INSPIRE].

[14] A. von Manteuffel and R.M. Schabinger, Quark and gluon form factors to four-loop order in QCD: the $N_{f}^{3}$ contributions, Phys. Rev. D 95 (2017) 034030 [arXiv:1611.00795] [INSPIRE].

[15] P. Maierhöfer, J. Usovitsch and P. Uwer, Kira - A Feynman integral reduction program, Comput. Phys. Commun. 230 (2018) 99 [arXiv:1705.05610] [InSPIRE].

[16] A.V. Smirnov and F.S. Chuharev, FIRE6: Feynman Integral REduction with Modular Arithmetic, arXiv: 1901.07808 [INSPIRE].

[17] T. Gehrmann and E. Remiddi, Differential equations for two loop four point functions, Nucl. Phys. B 580 (2000) 485 [hep-ph/9912329] [INSPIRE].

[18] R.N. Lee, Modern techniques of multiloop calculations, in Proceedings, 49th Rencontres de Moriond on QCD and High Energy Interactions, La Thuile, Italy, 22-29 March 2014, pp. 297-300 (2014) [arXiv:1405.5616] [INSPIRE].

[19] R.N. Lee and A.A. Pomeransky, Critical points and number of master integrals, JHEP 11 (2013) 165 [arXiv: 1308.6676] [INSPIRE].

[20] W. Chen, Reduction of Feynman Integrals in the Parametric Representation II: Reduction of Tensor Integrals, arXiv:1912.08606 [INSPIRE].

[21] R.P. Feynman, Space-time approach to quantum electrodynamics, Phys. Rev. 76 (1949) 769 [INSPIRE].

[22] N. Nakanishi, General Integral Formula of Perturbation Term in the Quantized Field Theory, Prog. Theor. Phys. 17 (1957) 401.

[23] K. Symanzik, Dispersion relations and vertex properties in perturbation theory, Prog. Theor. Phys. 20 (1958) 690.

[24] O.V. Tarasov, Connection between Feynman integrals having different values of the space-time dimension, Phys. Rev. D 54 (1996) 6479 [hep-th/9606018] [INSPIRE].

[25] T. Bitoun, C. Bogner, R.P. Klausen and E. Panzer, Feynman integral relations from parametric annihilators, Lett. Math. Phys. 109 (2019) 497 [arXiv:1712.09215] [INSPIRE].

[26] K.J. Larsen and Y. Zhang, Integration-by-parts reductions from unitarity cuts and algebraic geometry, Phys. Rev. D 93 (2016) 041701 [arXiv:1511.01071] [InSPIRE].

[27] J. Böhm, A. Georgoudis, K.J. Larsen, M. Schulze and Y. Zhang, Complete sets of logarithmic vector fields for integration-by-parts identities of Feynman integrals, Phys. Rev. D 98 (2018) 025023 [arXiv: 1712.09737] [INSPIRE].

[28] J. Böhm, A. Georgoudis, K.J. Larsen, H. Schönemann and Y. Zhang, Complete integration-by-parts reductions of the non-planar hexagon-box via module intersections, JHEP 09 (2018) 024 [arXiv: 1805.01873] [INSPIRE].

[29] R.N. Lee, LiteRed 1.4: a powerful tool for reduction of multiloop integrals, J. Phys. Conf. Ser. 523 (2014) 012059 [arXiv: 1310.1145] [INSPIRE].

[30] A.V. Smirnov and M.N. Tentyukov, Feynman Integral Evaluation by a Sector decomposiTion Approach (FIESTA), Comput. Phys. Commun. 180 (2009) 735 [arXiv:0807.4129] [INSPIRE].

[31] S. Actis, A. Ferroglia, G. Passarino, M. Passera and S. Uccirati, Two-loop tensor integrals in quantum field theory, Nucl. Phys. B 703 (2004) 3 [hep-ph/0402132] [INSPIRE]. 